\title{
Is the helium in the variable DB white dwarfs ${ }^{3} \mathrm{He}$ ? $^{\star}$
}

\author{
B. Wolff ${ }^{1,2}$, D. Koester ${ }^{1}$, M. H. Montgomery ${ }^{3}$, and D. E. Winget ${ }^{4}$ \\ 1 Institut für Theoretische Physik und Astrophysik, Universität Kiel, 24098 Kiel, Germany \\ e-mail: wolff, koester@astrophysik.uni-kiel.de \\ 2 European Southern Observatory, Karl-Schwarzschild-Str. 2, 85748 Garching bei München, Germany \\ 3 Institute of Astronomy, Madingley Road, Cambridge, CB3 0HA, UK \\ e-mail: mikemon@ast.cam.ac.uk \\ 4 Department of Astronomy, University of Texas at Austin, Austin, TX 78712, USA \\ e-mail: dew@astro.as.utexas.edu
}

Received 12 February 2002 / Accepted 29 March 2002

\begin{abstract}
Asteroseismological analysis of the Whole Earth Telescope observations of the prototype variable DB white dwarf GD358 indicates a possible composition interface at a depth of about $10^{-6} M_{\odot}$, which has usually been assumed to be the He/C transition zone. Such a small He layer is in contradiction with canonical evolution theory. Recently Montgomery \& Winget (2000) proposed that the transition zone is in fact the transition between the isotopes ${ }^{3} \mathrm{He}$ and ${ }^{4} \mathrm{He}$, which are separated by the same sedimentation process separating hydrogen and helium in the DA white dwarfs. Therefore, the visible surface helium should be ${ }^{3} \mathrm{He}$. We have tested this hypothesis spectroscopically on another variable DB using the known relative isotopic shifts of up to $0.5 \AA$. The spectra are consistent with a pure ${ }^{4} \mathrm{He}$ atmosphere, making a ${ }^{3} \mathrm{He} /{ }^{4} \mathrm{He}$ interface a very unlikely explanation for the mode spectrum of GD358.
\end{abstract}

Key words. stars: atmospheres - stars: interiors - stars: variables: general - stars: white dwarfs

\section{Introduction}

Variability in white dwarfs is due to non-radial pulsations, in many cases with many different modes simultaneously present, as identified by the indices of the spherical harmonics $l, m, k$ describing the distribution of amplitudes on the stellar surface and the radial node number in the interior.

The interpretation of observed modes and comparison with theoretical predictions has - under the name of asteroseismology - in the past decade become one of the most important tools to study the stellar interior and evolution, and after the exceptional case of our sun the white dwarfs have been the area of the most spectacular successes. Long and uninterrupted observing runs of at least 10 to 14 days are necessary to identify the many modes present. The main reason is the requirement to resolve almost degenerate modes split into triplets, quintuplets etc., by slow rotation or magnetic fields. This has led to the creation of the "Whole Earth Telescope" or WET (Nather et al. 1990; Winget et al. 1991), a network of observatories around the world joining for an observing campaign.

Send offprint requests to: D. Koester,

e-mail: koester@astrophysik.uni-kiel.de

* Based on observations collected at the European Southern Observatory, Paranal, Chile (ESO Programme 67.D-0047).
The comparison of the periods of identified modes with theoretical models allows us in principle to determine stellar parameters like mass, luminosity, rotation, and magnetic fields with very high accuracy. A special feature of the g-modes observed in white dwarfs (as opposed to p-modes in the sun) is the high sensitivity to the structure of the outer, non-degenerate layers. This is very fortunate, since this structure has been a matter of debate and controversy in the white dwarf community for almost 20 years. It is generally accepted that gravitational separation leads to an "onion shell" structure, with carbon/oxygen interior in most white dwarfs, surrounded by a helium shell and an outermost hydrogen shell (in the case of spectral type DA). Currently the balance seems again to be in favor of "thick layers", with about $10^{-2} M_{\odot}$ for the $\mathrm{He}$ and $10^{-4} M_{\odot}$ for the $\mathrm{H}$ shells, although some problems arguing e.g. for smaller $\mathrm{H}$ masses still exist. In the case of DB white dwarfs - with pure He atmospheres - the outer hydrogen layer has been completely lost, most likely during a late thermal pulse, and He as the next lightest element is floating on top.

These masses are the typical predictions of what we will call canonical stellar evolution calculations in this paper. By this we mean calculations like those of Iben \& Tutukov (1984) and Koester \& Schoenberner (1986), 
which include nuclear burning and mass loss during the final evolution from AGB to white dwarf, but no diffusion. The basic physical reason for these layer thicknesses is the extinction of shell-burning when the overlying layer is not heavy enough anymore to support the required temperature for $\mathrm{H}$ or $\mathrm{He}$ burning. Even including diffusion in a simplified approximation Iben \& MacDonald (1985) did not find a significant reduction in $\mathrm{H}$ layer thickness. All these calculations are relevant for DA white dwarfs, with a remaining hydrogen layer. There is as yet no completely consistent explanation for the DB white dwarfs, which agrees with all observational constraints, although Liebert et al. (1987) and many papers since have discussed the general ideas of diffusion and convective mixing in this context. Nevertheless, because of the general physics argument above, it is expected that the helium mass should be close to $10^{-2}$ of the total mass.

The variable DB or DBV stars are one of the three known groups of variable white dwarfs, with the prototype GD358. Its observation by the Whole Earth Telescope in May 1990 is one of the most spectacular WET successes, with the identification of more than 100 oscillation modes (Winget et al. 1994; Bradley \& Winget 1994). From a comparison of identified modes with theoretical pulsation models an accurate mass of $0.61 M_{\odot}$, a luminosity, differential rotation, and a weak magnetic field were deduced. One of the unexpected results was the determination of the thickness of the outer He layer of $10^{-5.7}$ of the stellar mass.

The physical reason this structure can be determined is that the interfaces between layers with different chemical compositions act as boundaries defining a cavity. If the wavelength of a mode is near a resonance with the size of such a cavity, the regular period spacing between modes of successive radial node number $k$ is perturbed.

GD358 was observed again by a WET campaign in May 1994, which showed remarkable differences in the power spectra compared to the 1990 campaign, and even during the observing run, which are not yet understood (Nather 1995). Metcalfe et al. (2000), using the same 1990 data as Bradley \& Winget (1994) but a different fit method based on a genetic algorithm found in a first analysis that also models with "thick" He layers of the order of $10^{-2}$ of the stellar mass - in agreement with theoretical expectations - could fit the data. Kawaler et al. (1999) have also questioned the conclusion on the differential rotation by demonstrating that a faster spinning core can explain the frequency splitting as well as a faster spinning outer envelope. It is therefore likely that the conclusions concerning the outer structure and layer thickness may have to be revised in the future. Nevertheless it is quite interesting to search for physical processes which could produce a chemical interface at a mass depth of $10^{-6}$.

Dehner \& Kawaler (1995) demonstrated that with some assumptions the inclusion of time-dependent diffusion can produce a model similar to that used by Bradley \& Winget (1994) to explain the GD358 WET results and suggested an evolutionary connection to the hot pulsating
Table 1. Isotope shifts of red ${ }^{3} \mathrm{HeI}$ lines compared to ${ }^{4} \mathrm{He}$ according to Fred et al. (1951).

\begin{tabular}{rr}
\hline \hline HeI line $(\AA)$ & shift for ${ }^{3}$ He in $\AA$ \\
\hline 3888 & 0.212 \\
3964 & 0.138 \\
4026 & 0.077 \\
4121 & 0.074 \\
5875 & 0.062 \\
6678 & 0.465 \\
7065 & 0.029 \\
7281 & 0.523 \\
\hline
\end{tabular}

pre-white dwarf PG1159 stars. Their models, however, did not have any hydrogen and thus would remain DB stars during their passage through the DB gap between 45000 and $28000 \mathrm{~K}$, contrary to observations.

A completely different proposal to solve the puzzle was presented by Montgomery \& Winget (2000). Basically this proposal takes gravitational separation seriously and to a consequence apparently overlooked until now: if no counteracting mechanism is at work, the separation should also work for the two isotopes of helium, with the lighter ${ }^{3} \mathrm{He}$ on top of ${ }^{4} \mathrm{He}$. Estimates for typical isotopic abundance ratios predict a ${ }^{3} \mathrm{He} /{ }^{4} \mathrm{He}$ interface at a depth of about $10^{-6}$ stellar masses, which would have the same effect on pulsation modes as the previously assumed $\mathrm{He} / \mathrm{C}$ interface, and the claim made is that this interface is the reason for the mode spectrum of GD358. Montgomery et al. (2001) discuss consequences and problems of this hypothesis and derive theoretical frequency spectra for such models, which give slightly improved fits to the observations.

From theoretical evolution calculations it is not expected that such an amount of ${ }^{3} \mathrm{He}$ could survive until the white dwarf stage. Galli et al. (1995) argue that in every stellar evolution from the main sequence in which hydrogen core burning is completed the remaining ${ }^{3} \mathrm{He}$ abundance should be vanishingly small. Thus, if the helium that is observed on the surface of the DBs is in fact that resulting from core hydrogen burning in the progenitor, no ${ }^{3} \mathrm{He}$ would be expected. However, as long as there is no consistent theory of the origin of the DBs we can hardly claim to understand the origin or composition of the helium layer. In such a case the best method to proceed is not theoretical speculation but a straightforward empirical test.

Fortunately this is possible since the spectral lines of ${ }^{3} \mathrm{He}$ are shifted relative to those of ${ }^{4} \mathrm{He}$ by different amounts of up to $0.5 \AA$ (Table 1, after Fred et al. 1951). The table lists four lines in the blue and four in the red part of the spectrum; each quartet has two triplet lines with small and two singlet lines with larger isotopic shift. Since the stellar space velocity cannot be determined 
independently, this relative isotopic shift is the only way to determine isotope abundances; with relative shifts of about $0.5 \AA$ in the red the effect should be easily measurable on high resolution high $S / N$ spectra.

The direct test of the gravitational settling hypothesis would be the spectroscopy of GD358 itself. However, this northern object was not accessible to us with a large telescope and high-resolution spectrograph. We have therefore selected the brightest variable DB on the southern sky EC 20058-5234 (Koen et al. 1995) - as our target. This assumes that the variable DBs form a homogenous group and that results on the chemical composition of one group member should be applicable to all.

\section{Observations and data reduction}

We have observed five stars with the Kueyen (UT2) telescope of the ESO Very Large Telescope (VLT) during one night in June 2001: the variable DB white dwarf EC 20058-5234 (WD 2006-523), the non-variable DB L 204-118 (WD 1726-578), and as comparison objects the $\mathrm{sdO}$ star $\mathrm{LSIV}+10^{\circ} 9$, the main sequence $\mathrm{B}$ star $\mathrm{BD}-13^{\circ} 4937$ in the open cluster NGC6611, and the extreme helium star $\mathrm{LSIV}+6^{\circ} 2$. The spectra were taken with the UV-Visual Echelle Spectrograph (UVES). This instrument provides high-resolution spectroscopy in the range from about 3000 to $11000 \AA$. We have selected the DICHROIC1 mode of UVES with the central wavelengths $3900 \AA$ and $6500 \AA$ to observe the blue and red parts of the spectra simultaneously. With a slit width of 0.7 , a resolution of 55000 in the blue and 60000 in the red is obtained.

The blue arm of UVES is equipped with one EEV CCD detector of $2048 \times 3000$ pixel resolution; the red arm with a mosaic of one EEV and one MIT-LL detector, each with $2048 \times 4096$ pixels. The CCDs were read out in fast readout mode with low gain and $1 \times 1$ binning.

Table 2 contains an overview of the observations. The exposure times were chosen to obtain signal-to-noise ratios of about 50 in the continuum. Long exposures were split into smaller exposures of one hour. A wavelength calibration with a ThAr lamp was carried out after each exposure. For each detector, five bias and lamp flatfield images were obtained at the end of the night.

The data have been reduced with routines of the 'echelle' package within IRAF. The bias and flatfield images were averaged, and each science exposure has been corrected with these images. The individual echelle orders of the object spectra and the associated ThAr calibrations have been extracted with the same parameter settings. A wavelength solution has been established with the use of the ThAr line atlas provided by the ESO homepage. The root mean square deviation between our solution and the catalog wavelengths is $0.01 \AA$. After calibrating the wavelengths, individual spectra of the same object have been averaged. Finally, all echelle orders were rebinned on a common wavelength scale with $\Delta \lambda=0.02 \AA$ and were merged into single spectra. In Fig. 1 we present the
Table 2. Log of VLT/UVES observations.

\begin{tabular}{lllc}
\hline \hline Object & Type & Date and time (UT) & Exp.T. \\
\hline L 204-118 & DB & 2001-06-30T22:58:05 & $3600 \mathrm{~s}$ \\
L 204-118 & DB & 2001-07-01T00:10:41 & $3600 \mathrm{~s}$ \\
L 204-118 & DB & 2001-07-01T01:23:22 & $3600 \mathrm{~s}$ \\
L 204-118 & DB & 2001-07-01T02:29:45 & $3600 \mathrm{~s}$ \\
LS IV +6 2 & EHe & 2001-07-01T03:36:19 & $1200 \mathrm{~s}$ \\
BD -13 4937 & B2V & 2001-07-01T04:13:31 & $300 \mathrm{~s}$ \\
EC 20058-5234 & DBV & 2001-07-01T04:27:29 & $3600 \mathrm{~s}$ \\
EC 20058-5234 & DBV & 2001-07-01T05:37:36 & $3600 \mathrm{~s}$ \\
EC 20058-5234 & DBV & 2001-07-01T06:43:21 & $3600 \mathrm{~s}$ \\
EC 20058-5234 & DBV & 2001-07-01T07:50:02 & $3600 \mathrm{~s}$ \\
LS IV +10 9 & sdO & 2001-07-01T09:36:27 & $1500 \mathrm{~s}$ \\
\hline
\end{tabular}

observations of the four red He I lines, which are the most important lines for our analysis.

We have tested the wavelength calibration using the $\mathrm{O}_{2}(\approx 6870-6910 \AA)$ and $\mathrm{H}_{2} \mathrm{O}(\approx 7230-7290 \AA)$ lines of the earth's atmosphere, which are visible in the red part of the spectra. The observed wavelengths have been measured by fitting Voigt profiles to the lines in the spectra of EC 20058-5234 and L 204-118. The theoretical values have been obtained from the HITRAN2000 database of molecular wavelengths (Rothman et al. 1998). The vacuum wavelengths from this database have been transfered into air wavelengths using the formula given by Cowley et al. (2000).

The wavelengths of the $\mathrm{H}_{2} \mathrm{O}$ lines deviate on average by $0.005 \AA$ in both observations; the root mean square deviation is $0.012 \AA$. For the $\mathrm{O}_{2}$ lines, the deviations go more or less in the same direction with an average of $0.021 \AA$ and an rms of $0.022 \AA$. We conclude from these comparisons that the wavelength calibration is correct within $0.02 \AA$. This agrees with the binning of the final spectra.

\section{Analysis}

We started the analysis by fitting Voigt profiles to the line cores to measure the wavelengths of the observed HeI lines. This method was successful for the variable DB EC 20058-5234 and for the comparison stars $\mathrm{LS} \mathrm{IV}+6^{\circ} 2$ and LS IV $+10^{\circ} 9$. The non-variable DB L 204118 exhibits asymmetrical line profiles which prevent the use of simple profiles. The main sequence B star $\mathrm{BD}-13^{\circ} 4937$ has rotationally broadened lines which do not allow accurate measurements.

In order to compare the observed wavelengths to the laboratory values we have also measured the latter from a model spectrum. This procedure takes into account small asymmetries and shifts from the Stark broadening of the He lines as well as the fact that the lines usually consist of several discrete components which cannot be separated 

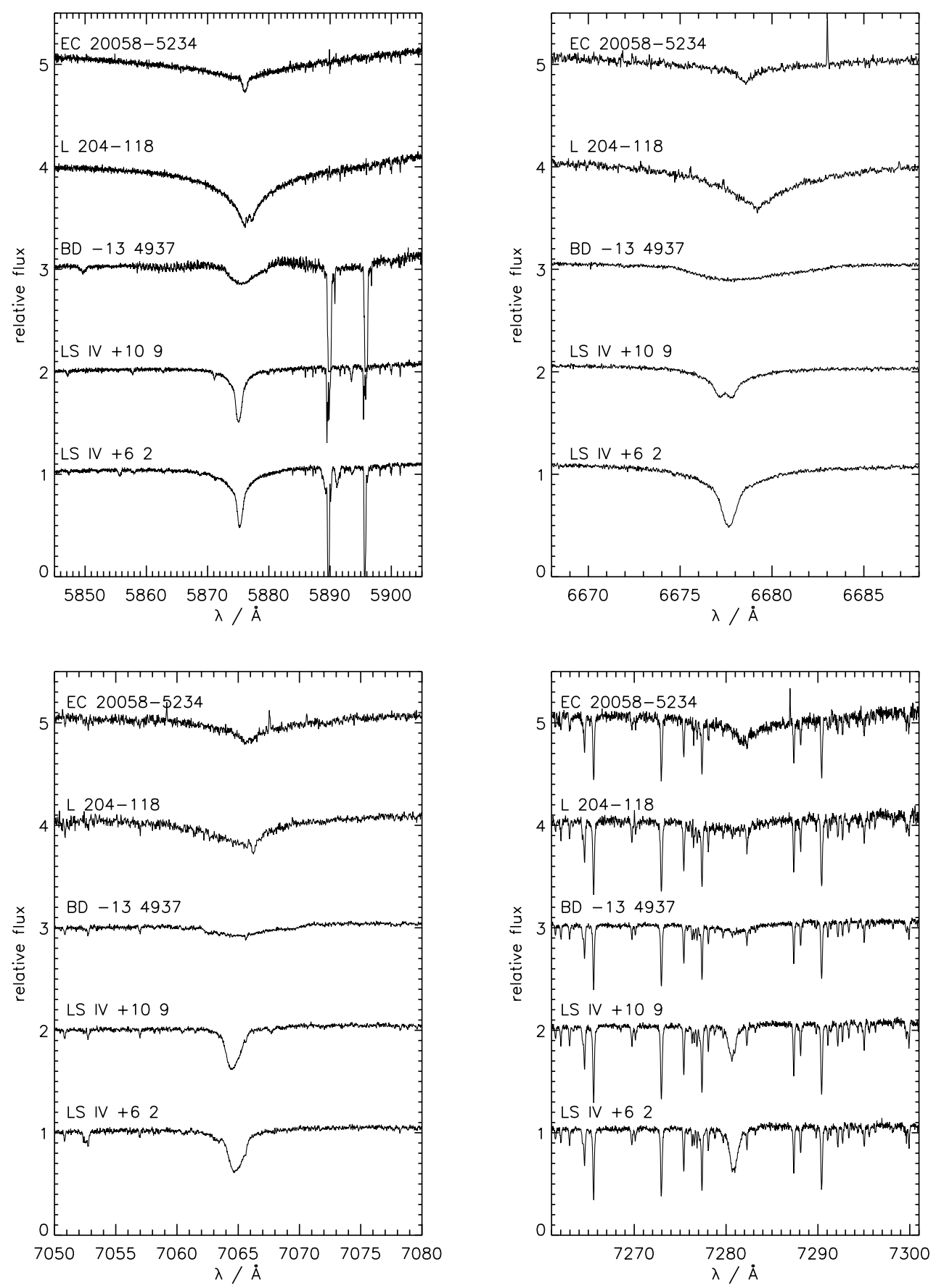

Fig. 1. Observations of the four red HeI lines (5875, 6678, 7065, and $7281 \AA$ ).

at our resolution. It ensures that the same method is used to determine observed and theoretical wavelengths.

As already explained in the introduction the observed wavelengths cannot be directly compared to the laboratory values because of the unknown space velocity of the objects. This adds a shift $\Delta \lambda=(v / c) \lambda$ (with the velocity of light $c$ ) to each line. Because these shifts depend on $\lambda$ for a constant space velocity $v$ it is also not straightforward to compare the wavelength differences between pairs of lines. The most direct method is to calculate space velocities from each line and test whether the result is compatible with a constant value for either ${ }^{3} \mathrm{He}$ or ${ }^{4} \mathrm{He}$.
Table 3 shows the results for the four strong He I lines which can be observed in the red part of the spectra. These four lines provide the most stringent test for the presence of ${ }^{3} \mathrm{He}$ since the lines at $6678 \AA$ and $7281 \AA$ have the strongest isotope shifts of ${ }^{3} \mathrm{He}$ compared to ${ }^{4} \mathrm{He}(\Delta \lambda \approx$ $0.5 \AA$ or $\approx 20 \mathrm{~km} \mathrm{~s}^{-1}$ ) whereas the other two lines have negligible shifts.

All four velocity measurements for each star are very similar. Especially the lines at $6678 \AA$ and $7281 \AA$ do not exhibit different velocities which would have been expected if the atmosphere predominantly consisted of ${ }^{3}$ He. Therefore, the two comparison stars 
Table 3. Apparent velocities as derived from the differences of the observed HeI lines to the laboratory wavelengths of ${ }^{4} \mathrm{He}$. The wavelengths have been measured using Voigt profiles.

\begin{tabular}{llll}
\hline \hline He I line & $\begin{array}{l}\text { EC 20058-5234 } \\
v / \mathrm{km} \mathrm{s}^{-1}\end{array}$ & $\begin{array}{l}\mathrm{LS} \mathrm{IV}+6^{\circ} 2 \\
v / \mathrm{km} \mathrm{s}^{-1}\end{array}$ & $\begin{array}{l}\mathrm{LS} \mathrm{IV+10} 9 \\
v / \mathrm{km} \mathrm{s}^{-1}\end{array}$ \\
\hline 5875 & 16.33 & -25.00 & -34.69 \\
6678 & 17.06 & -24.69 & -32.32 \\
7065 & 18.67 & -22.49 & -32.67 \\
7281 & 16.88 & -28.41 & -36.64 \\
\hline
\end{tabular}

as well as EC 20058-5234 seem to have nearly pure ${ }^{4} \mathrm{He}$ atmospheres.

The simple fit using Voigt profiles does not provide an estimate of the uncertainties of the measurements. We have, therefore, fitted synthetic spectra to the observed lines and have determined the shifts in wavelengths by a $\chi^{2}$ analysis. The models used were LTE atmospheres with a pure ${ }^{4} \mathrm{He}$ composition, with pure ${ }^{3} \mathrm{He}$, or with a mixture of ${ }^{3} \mathrm{He}$ and ${ }^{4} \mathrm{He}$. For a description of our methods to calculate theoretical atmospheres and synthetic spectra we refer to the paper of Finley et al. (1997).

The velocity shifts have been measured from each helium line separately. The model spectra have been fitted to the observation at two regions near the respective line. The $\chi^{2}$ values for several velocity shifts have been calculated from a small region near the line center, including typically $N \approx 100$ wavelength bins. From the $\chi^{2}$ values, the best fitting velocity and a $1 \sigma$ error have been calculated. We have quadratically added an additional uncertainty of $0.02 \AA$ to account for systematic errors of the wavelength calibration.

Table 4 shows the results for EC 20058-5234 with a model atmosphere of $T_{\text {eff }}=24000 \mathrm{~K}, \log g=8.0$, and pure ${ }^{3} \mathrm{He}$ and ${ }^{4} \mathrm{He}$ compositions, respectively. In addition to the four lines in the red part of the spectrum, the four most prominent blue lines are also added.

The four red lines have velocities similar to the values determined using Voigt profiles. The deviations are of the order of $1 \mathrm{~km} \mathrm{~s}^{-1}$ and demonstrate the typical uncertainty of the profile fitting process. Nevertheless, the velocities derived from the red lines and a pure ${ }^{4} \mathrm{He}$ atmosphere are very similar. The blue lines show somewhat larger deviations; apparently the agreement between the model lines and the complex observed profiles is less satisfactory than in the red part of the spectrum. The strongest deviations, however, are not correlated with the largest expected isotope shifts.

The overall agreement of the velocities from different lines is destroyed if a pure ${ }^{3} \mathrm{He}$ atmosphere is used as reference. This can be very convincingly seen from the lines at $6678 \AA$ and $7281 \AA$, which have the strongest isotope shifts. Therefore, an atmosphere consisting only of ${ }^{3} \mathrm{He}$ can be ruled out.
Table 4. Results from the analysis with template spectra: apparent velocities of the HeI lines in the spectrum of EC 20058-5234 as compared to model spectra with pure ${ }^{4} \mathrm{He}$ and pure ${ }^{3} \mathrm{He}$, respectively.

\begin{tabular}{lcr}
\hline \hline & $\begin{array}{c}{ }^{4} \mathrm{He} \\
v / \mathrm{km} \mathrm{s}^{-1}\end{array}$ & \multicolumn{1}{c}{$\begin{array}{c}{ }^{3} \mathrm{He} \\
v / \mathrm{km} \mathrm{s}^{-1}\end{array}$} \\
\hline 3888 & $19.45 \pm 2.20$ & $2.67 \pm 2.22$ \\
3964 & $24.08 \pm 2.12$ & $10.25 \pm 2.11$ \\
4026 & $21.29 \pm 6.33$ & $17.52 \pm 4.62$ \\
4121 & $18.77 \pm 1.88$ & $13.42 \pm 2.09$ \\
5875 & $17.01 \pm 1.95$ & $14.91 \pm 1.97$ \\
6678 & $16.21 \pm 1.64$ & $-5.91 \pm 1.70$ \\
7065 & $18.06 \pm 1.79$ & $18.77 \pm 1.81$ \\
7281 & $15.80 \pm 1.81$ & $-7.22 \pm 1.88$ \\
\hline
\end{tabular}

The weighted mean from the ${ }^{4} \mathrm{He}$ fits of the red lines is $\bar{v}=16.74 \pm 0.89 \mathrm{~km} \mathrm{~s}^{-1}$; the error is propagated from the errors of the individual lines. In order to test quantitatively the hypothesis that a pure ${ }^{4} \mathrm{He}$ atmosphere is a valid description of the observations, i.e. that the derived velocities are compatible with the velocity $\bar{v}$, we have calculated the value $\chi^{2}=\sum\left(v_{i}-\bar{v}\right)^{2} / \sigma_{i}^{2}$ from these four lines. The reduced $\chi^{2}$ is $\chi_{\mathrm{r}}^{2}=\chi^{2} /(n-1)=0.31(n=4$, one parameter). This is equivalent to the probability of $P\left(>\chi^{2}\right)=0.82$ that a larger $\chi^{2}$ value than the actual one will be found in a measurement by chance if the measured velocities are normally distributed.

From the ${ }^{3} \mathrm{He}$ fits we derive $\bar{v}=4.60 \pm 0.92$ with $\chi_{\mathrm{r}}^{2}=$ 55.48 and $P\left(>\chi^{2}\right)=7.51 \times 10^{-36}$. The ${ }^{3}$ He solution is, therefore, extremely improbable. On the other hand, the large probability of the ${ }^{4} \mathrm{He}$ solution may indicate that the size of the errors is slightly overestimated.

Since an atmosphere consisting of ${ }^{3} \mathrm{He}$ only can be ruled out it is also worthwhile to examine mixtures of the two helium isotopes. We have calculated $\chi^{2}$ values from fits of model atmospheres with ${ }^{3} \mathrm{He} /\left({ }^{3} \mathrm{He}+{ }^{4} \mathrm{He}\right)=0.0$, $0.25,0.5,0.75$, and 1.0 to the central regions of the observed four lines in the red spectrum. For each fit, we have assumed a single velocity, which is determined from the line at $7065 \AA$, for all lines. The error of the observed flux is set equal to the root mean square deviation of the fit with ${ }^{3} \mathrm{He} /\left({ }^{3} \mathrm{He}+{ }^{4} \mathrm{He}\right)=0.0$.

The $\chi^{2}$ values of the fits show, as expected, a minimum at ${ }^{3} \mathrm{He} /\left({ }^{3} \mathrm{He}+{ }^{4} \mathrm{He}\right)=0.0$ and monotonically increase towards ${ }^{3} \mathrm{He} /\left({ }^{3} \mathrm{He}+{ }^{4} \mathrm{He}\right)=1.0$. We have fitted a parabola to the three lowest values and determined from it a formal solution of ${ }^{3} \mathrm{He} /\left({ }^{3} \mathrm{He}+{ }^{4} \mathrm{He}\right)=-0.01 \pm 0.26$ on a $3 \sigma$ confidence level $\left(\Delta \chi^{2}=9\right)$. We finally conclude that mixtures with ${ }^{3} \mathrm{He} /\left({ }^{3} \mathrm{He}+{ }^{4} \mathrm{He}\right)>0.27$ can be ruled out.

Originally we had intended to use the non-variable DB L 204-118 as an additional comparison object. However, the effective temperature is much lower than that of EC 20058-5234 (in the range of 12 000-14000 K, depending on which lines are used for a comparison with 
theoretical models). The lines are not fitted well by our current models, most likely because these do not take into account van der Waals broadening by neutral helium, which should be significant at these temperatures. Moreover, the important line $5875 \AA$, which shows a much deeper line core than the LTE models already in EC 20058-5234, shows a complex structure in L 204-118, possibly with a small emission core. We assume that these structures may be caused by NLTE effects, similar to the NLTE effects in the cores of $\mathrm{H} \alpha$ in DA stars. While we have not found any obvious evidence for ${ }^{3} \mathrm{He}$ in L 204-118, the measurements are too uncertain to be useful.

\section{Discussion}

The results of our study rule out the existence of a pure ${ }^{3} \mathrm{He}$ layer on top of ${ }^{4} \mathrm{He}$ in the variable $\mathrm{DB}$ white dwarf EC 20058-5234. The upper limit on the ${ }^{3} \mathrm{He} /\left({ }^{3} \mathrm{He}+{ }^{4} \mathrm{He}\right)$ abundance is not very stringent with about $27 \%$. However, if gravitational settling is assumed as the origin for such peculiar composition, anything but a pure layer - either ${ }^{3} \mathrm{He}$ or ${ }^{4} \mathrm{He}$ - would be very difficult to understand. There is not yet available any pulsational study comparable to the work on the prototype GD358, and our result is not directly applicable to that object, where the "thin" layer and compositional interface at a depth of $2 \times 10^{-6}$ of the total mass has been claimed. However, the similarity of the variable DBs as a group makes it highly unlikely, that such a separation of isotopes could work in GD358. Our results lend support to the expectation from stellar evolutionary theory (see e.g. Galli et al. 1995) that most of the original ${ }^{3} \mathrm{He}$ is destroyed in the core of the progenitor stars. The interpretation of the complex frequency spectrum of GD358 and other variable DB may lead to a different solution for the outer structure, as already indicated in the recent work by Metcalfe et al. (2000).

Our results can also be used to draw some conclusions in the broader context of white dwarf spectral evolution. The standard explanation for the metal traces found in about $20 \%$ of cool He-rich white dwarfs currently is a combination of accretion from interstellar matter with subsequent gravitational settling as discussed in great detail by Dupuis et al. (1992, 1993a,b). There is, however, the unsolved problem, how He-rich objects like the DBs of this study can survive in the presence of accretion. One of the mechanisms proposed to prevent the accretion of hydrogen, while allowing that of heavier elements, goes back to Michaud \& Fontaine (1979). This is the hot corona model, where the electric field of the He-dominated atmosphere prevents protons from accreting but not metals. Our constraints on the abundance or rather absence of ${ }^{3} \mathrm{He}$ allows to derive another constraint on the amount of accreted matter. In a helium envelope convection would keep ${ }^{3} \mathrm{He}$ and ${ }^{4} \mathrm{He}$ mixed in the upper layers up to the photosphere (in a DB with $24000 \mathrm{~K}$ effective temperature the convection zone reaches up to optical depths $\sim 0.01$ ); gravitational separation would occur below the bottom of the convection zone only. The total depth of the convection zones in the range of variable DBs varies between $10^{-11}$ to $10^{-14}$ of the stellar mass, depending on $T_{\text {eff }}$ and the version of the convection theory used (e.g. Benvenuto \& Althaus 1997, Fig. 10). This mass is not pure ${ }^{3} \mathrm{He}$ according to our results. Assuming a ${ }^{3} \mathrm{He} /{ }^{4} \mathrm{He}$ ratio of about $2 \times 10^{-4}$ for the ISM (Gloeckler \& Geiss 1996), the total amount of accreted He must therefore be smaller than $3 \times 10^{-8}$ to $3 \times 10^{-11} M_{\odot}$ for a typical DB of $0.6 M_{\odot}$; the upper limit for the total accreted mass - assuming solar abundances - would be larger by a factor of 10. This is another observational constraint for the accretion/diffusion scenario; it is, however, beyond the scope of this work to study the consequences in more detail.

Acknowledgements. This work has been supported by the Deutsches Zentrum für Luft- und Raumfahrt (DLR) under grant 50 OR 96173.

\section{References}

Benvenuto, O. G., \& Althaus, L. G. 1997, MNRAS, 288, 1004 Bradley, P. A., \& Winget, D. E. 1994, ApJ, 421, 236

Cowley, C., Wiese, W. L., Fuhr, J., \& Kuznetsova, L. A. 2000, in Allen's Astrophysical Quantities, ed. A. N. Cox, 4th edition (Springer, New York), 69

Dehner, B. T., \& Kawaler, S. D. 1995, ApJ, 445, L141

Dupuis, J., Fontaine, G., Pelletier, C., \& Wesemael, F. 1992, ApJS, 82, 505

Dupuis, J., Fontaine, G., Pelletier, C., \& Wesemael, F. 1993a, ApJS, 84, 73

Dupuis, J., Fontaine, G., \& Wesemael, F. 1993b, ApJS, 87, 345

Finley, D. S., Koester, D., \& Basri, G. 1997, ApJ, 488, 375

Fred, M., Tomkins, F. S., Brody, J. K., \& Hamermesh, M. 1951, Phys. Rev., 82, 406

Galli, D., Palla, F., Ferrini, F., \& Penco, U. 1995, ApJ, 443, 536

Gloeckler, G., \& Geiss, J. 1996, Nature, 381, 210

Iben, I., \& MacDonald, J. 1985, ApJ, 296, 540

Iben, I., \& Tutukov, A. V. 1984, ApJ, 282, 615

Kawaler, S. D., Sekii, T., \& Gough, D. 1999, ApJ, 516, 349

Koen, C., O’Donoghue, D., Stobie, R. S., Kilkenny, D., \& Ashley, R. 1995, MNRAS, 277, 913

Koester, D., \& Schoenberner, D. 1986, A\&A, 154, 125

Liebert, J., Fontaine, G., \& Wesemael, F. 1987, Mem. della Soc. Astron. Ital., 58, 17

Metcalfe, T. S., Nather, R. E., \& Winget, D. E. 2000, ApJ, 545,974

Michaud, G., \& Fontaine, G. 1979, ApJ, 229, 694

Montgomery, M. H., Metcalfe, T. S., \& Winget, D. E. 2001, ApJ, 548, L53

Montgomery, M. H., \& Winget, D. E. 2000, Baltic Astron., 9, 23

Nather, R. E. 1995, Baltic Astron., 4, 117

Nather, R. E., Winget, D. E., Clemens, J. C., Hansen, C. J., \& Hine, B. P. 1990, ApJ, 361, 309

Rothman, L. S., Rinsland, C. P., Goldman, A., et al. 1998, J. Quant. Spectrosc. Rad. Transfer, 60, 665

Winget, D. E., Nather, R. E., Clemens, J. C., et al. 1991, ApJ, 378,326

Winget, D. E., Nather, R. E., Clemens, J. C., et al. 1994, ApJ, 430,839 